\title{
Rutin promotes the formation and osteogenic differentiation of human periodontal ligament stem cell sheets in vitro
}

\author{
BIN ZHAO $^{1,2}$, YUNPENG ZHANG ${ }^{1,2}$, YIXUAN XIONG ${ }^{1,2}$ and $\mathrm{XIN} \mathrm{XU}^{1,2}$ \\ ${ }^{1}$ School of Stomatology, Shandong University; ${ }^{2}$ Shandong Provincial Key Laboratory of \\ Oral Tissue Regeneration, Jinan, Shandong 250012, P.R. China
}

Received July 5, 2019; Accepted October 15, 2019

DOI: $10.3892 /$ ijmm.2019.4384

\begin{abstract}
Cell sheet technology is a novel tissue engineering technology that has been rapidly developed in recent years. As a novel technology, cell sheet technology is expected to become one of the preferred methods for cell transplantation. The present study investigated the biological effects of rutin on the formation of periodontal ligament stem cell (PDLSC) sheets and their resultant osteogenic properties. The results of Cell Counting Kit-8 (CCK-8) assay demonstrated that a concentration of $1 \times 10^{-6} \mathrm{~mol} / \mathrm{l}$ rutin promoted the proliferation of PDLSCs more effectively compared with other designed concentrations. Rutin-modified cell sheets could be induced by complete medium supplemented with $20 \mu \mathrm{g} / \mathrm{ml}$ vitamin $\mathrm{C}$ (VC) and $1 \times 10^{-6} \mathrm{~mol} / \mathrm{l}$ rutin. Rutin-modified cell sheets appeared thicker and more compact compared with the VC-induced PDLSC sheets, demonstrating more layers of cells ( 3 or 4 layers), which secreted a richer extracellular matrix (ECM). Furthermore, the improved cell sheets exhibited varying degrees of increases in the mRNA and protein expression of collagen type I (COL1), alkaline phosphatase (ALP), runt-related transcription factor 2 (RUNX2) and osteopontin (OPN). Combined treatment with $\mathrm{VC}$ and rutin promoted the formation of PDLSC sheets and enhanced the osteogenic differentiation potential of the cell sheets. Therefore, rutin-modified cell sheets of PDLSCs are expected to play an important role in the treatment of periodontal tissue regeneration by stem cells.
\end{abstract}

\section{Introduction}

The loss of periodontal support tissue caused by periodontitis and other factors is the primary cause of tooth loss in adults (1). The treatment of periodontitis and the restoration of periodontal defects are key issues that require improvements. Although

Correspondence to: Dr Xin Xu, School of Stomatology, Shandong University, 44-1 Wenhua Xi Road, Jinan, Shandong 250012, P.R. China E-mail: xinxu@sdu.edu.cn

Key words: rutin, periodontal ligament, stem cells, osteogenic differentiation common periodontal treatments, such as scaling, curettage and flap surgery can control the progression of periodontitis and prevent the further loss of periodontal support tissue, they cannot regenerate the lost tissue effectively (2). The rapid development of tissue engineering research brings new hope to the treatment of periodontitis and makes it possible to regenerate periodontal tissue by using the methods of tissue engineering (3). Cell sheet technology is a novel tissue engineering technology that has rapidly developed in recent years (4). Cell sheets play a role in maintaining the biological characteristics and performing the functions of stem cells (5-7). Studies have demonstrated that cell sheets have more potent tissue regenerative effects in the myocardium, liver, cornea, bone tissue and periodontal regeneration compared with other tissue engineering methods (8-12). Cell sheets were originally obtained from a thermosensitive material culture dish invented by Okano et al, and they were separated from each other by temperature changes (13). A previous study demonstrated that cell sheet technology can preserve the extracellular matrix (ECM) and the connection between cells in vitro, thus avoiding the application of exogenous biomaterials and improving the survival rate of cell transplantation (14). Traditional temperature-sensitive cell culture dishes are complex to prepare and expensive to equip. Good biocompatibility, operability, quantifiable control and adjustment of the size and thickness of the sheets could make cell sheet technology one of the preferred methods for cell transplantation.

In recent years, a number of improved methods have been developed to make it easier to obtain biologically active cell sheets. It has been shown that cell sheets obtained with dexamethasone and vitamin $\mathrm{C}$ (VC) can promote bone formation (15). VC, a cofactor in the biological reaction of the whole body, is an important nutrient for human health (16). VC participates in the synthesis and function of immune factors, as well as collagen synthesis (17). Studies have demonstrated that $\mathrm{VC}$ is essential for the biosynthesis of ECM and can stimulate the proliferation and differentiation of stem cells in vitro by mimicking the biological environment $(18,19)$. In addition, a method of obtaining cell sheets by VC induction culture was reported in 2012, which rendered the preparation of cell sheets simpler (20). Compared with traditional thermosensitive materials, it is easier to obtain complete cell sheets with VC induction (14,20,21). For the acceleration of the clinical application of periodontal tissue engineering, the 
further investigation of safe, effective and simple cell sheet technology is necessary.

It has been demonstrated that bioflavonoids exhibit a variety of biological activities (22), which are expected to be substitutes for growth factors for the in vitro regulation of cell biological properties. Rutin is a natural bioflavonoid that is widely present in plants (23). Rutin exerts antioxidant and anti-free radical effects, and can be used in the treatment of cardiovascular and cerebrovascular diseases, tumors and inflammation (24-26). As a common bioflavonoid, rutin is inexpensive, safe and easy to obtain $(27,28)$. In vitro, experiments have identified that rutin can promote cell proliferation and osteogenic differentiation, which can effectively prevent and treat osteoporosis (29). Rutin, a type of vitamin $\mathrm{P}$, is a glycoside of dehydro flavanone and coexists with VC in food. Vitamin P is a hydrogen transmitter, which can prevent $\mathrm{VC}$ from being oxidized and can enhance the effects of VC (30-33). Based on the aforementioned studies, the present study proposed the hypothesis that the addition of rutin and $\mathrm{VC}$ during the preparation of cell sheets could promote the formation of cell sheets and improve their osteogenic properties.

The present study investigated the effects of rutin on the formation, proliferation and osteogenic differentiation of periodontal ligament stem cell (PDLSC) sheets, and provided a theoretical basis for the improvement of cell sheet technology, which may accelerate the clinical transformation of periodontal cell therapy.

\section{Materials and methods}

Cell culture. The preparation and culture of PDLSCs was performed according to previous studies (34-36). All the schemes dealing with human periodontal tissues were approved by the Ethics Committee of Shandong University (Shandong, China). Informed consent was obtained in writing by all donors and their parents. Healthy premolars extracted due to orthodontic reasons of adolescents aged 12-16 years ( 3 boys and 3 girls, the boys were 12, 14 and 15 years old, and the girls were 13, 16 and 16 years old) were selected in May, 2018. Periodontal ligament tissues of the middle and lower part of the root were scraped and cut into small sections using a surgical knife. The sections were digested in $\alpha$-minimum essential medium ( $\alpha$-MEM; Gibco, Thermo Fisher Scientific, Inc.) containing $1 \%$ collagenase (Sigma-Aldrich; Merck $\mathrm{KGaA}$ ) and $1 \%$ dispase (Sigma-Aldrich; Merck KGaA) for $60 \mathrm{~min}$ at $37^{\circ} \mathrm{C}$. Following digestion, the tissue was filtered through a $70-\mu \mathrm{m}$ filter to obtain suspended single cells. The obtained cells were inoculated in a flask of $25 \mathrm{~cm}^{2}$ and then cultured in $\alpha$-MEM containing $10 \%$ fetal bovine serum (FBS; Gibco, Thermo Fisher Scientific, Inc.), $100 \mathrm{U} / \mathrm{ml}$ penicillin (Beyotime, Institute of Biotechnology) and $100 \mathrm{mg} / \mathrm{ml}$ streptomycin (Beyotime Institute of Biotechnology) at $37^{\circ} \mathrm{C}$ in $5 \% \mathrm{CO}_{2}$.

Flow cytometric identification of cell surface markers. The BD StemflowTM hMSC Analysis kit (BD Biosciences) was used to identify the immunophenotype. First generation cells were cultured in a culture dish $(10 \times 1 \times 10 \mathrm{~cm})$. When the cell density reached $90 \%$, the cells were washed with PBS (Corning, Inc.) twice and digested by trypsinase and then a single cell suspen- sion was prepared. The cells were then separated into sterile tubes. A mesenchymal stem cell (MSC)-positive cocktail (CD90 FITC, CD105 PerCP-Cy5.5, CD73 APC, CD44) and MSC-negative cocktail (CD34, CD11b, CD19, CD45, HLA-DR) were added to the tubes in the dark at $4^{\circ} \mathrm{C}$ for $20 \mathrm{~min}$. After the cells were identified by flow cytometry (BD Biosciences), PDLSCs were passaged to the third generation, and cells of this generation were used in the subsequent experiments.

Multiple differentiation analysis. PDLSCs of the third generation were inoculated into 6-well plates at a density of $1 \times 10^{5} /$ well. When the cells were attached, the medium was replaced with osteogenic induction medium, namely $\alpha$-MEM containing $10 \% \mathrm{FBS}, 10 \mathrm{nmol} / \mathrm{l}$ dexamethasone (Beijing Solarbio Science \& Technology Co., Ltd.), $50 \mu \mathrm{g} / \mathrm{ml} \mathrm{VC} \mathrm{(Sigma,} \mathrm{Aldrich;} \mathrm{Merck} \mathrm{KGaA)} \mathrm{and} 10 \mathrm{mmol} / 1$ $\beta$-glycerophosphate (Beijing Solarbio Science \& Technology Co., Ltd.). Following 4 weeks of induction, the cells were fixed with $4 \%$ paraformaldehyde for $30 \mathrm{~min}$, then stained with Alizarin Red (Sigma-Aldrich; Merck KGaA) for $15 \mathrm{~min}$ at room temperature and rinsed with PBS 3 times. The nodules were observed under an inverted microscope (Olympus Corp.) and images were obtained. Likewise, for adipogenic induction, induction was carried out with an adipogenic induction solution, which included $\alpha$-MEM containing $10 \%$ FBS, $2 \mu \mathrm{M}$ dexamethasone, $0.2 \mathrm{mM}$ indomethacin (Sigma-Aldrich; Merck $\mathrm{KGaA}$ ), $0.01 \mathrm{mg} / \mathrm{ml}$ insulin (Sigma-Aldrich; Merck KGaA) and $0.5 \mathrm{mM}$ 3-isobutyl-1-methylxanthine (Sigma-Aldrich; Merck KGaA). The medium was changed every 3 days, after which the induction and fixation steps were performed, and $0.3 \%$ Oil Red O (Cyagen Biosciences) staining was used for $30 \mathrm{~min}$ at room temperature. The adipogenic droplets were then observed under an inverted microscope (IX73; Olympus Corp.).

Detection of cell proliferative activity. The Cell Counting Kit-8 (CCK-8; Dojindo Molecular Technologies, Inc.) was used to detect the cell proliferative activity. In total, 2,000 cells/well were seeded into a 96-well culture plate and divided into 6 groups with 5 multiple wells in each group. Cell proliferation was continuously examined for 3 days, and the culture medium was changed every day. After the cells had attached, PDLSCs were cultured with various concentrations of rutin $\left(0,1 \times 10^{-8}\right.$, $1 \times 10^{-7}, 1 \times 10^{-6}, 1 \times 10^{-5}$ and $\left.1 \times 10^{-4} \mathrm{~mol} / \mathrm{l}\right)$ in $\alpha$-MEM containing a certain concentration of $\mathrm{VC}(20 \mu \mathrm{g} / \mathrm{ml})$. At the indicated time point, each well was incubated with $10 \mu \mathrm{l}$ CCK-8 solution for $1 \mathrm{~h}$. The absorbance was measured at $450 \mathrm{~nm}$ by a microplate reader (SPECTROstar Nano).

Formation and observation of cell sheets. A total of $1 \times 10^{5}$ cells per well were seeded into a 6 -well plate. Following cell attachment, $20 \mu \mathrm{g} / \mathrm{ml} \mathrm{VC}$ and $1 \times 10^{-6} \mathrm{~mol} / 1$ rutin were added to the medium as the experimental group. In addition, $20 \mu \mathrm{g} / \mathrm{ml} \mathrm{VC}$ were added to the medium in the control group. Following 10 days of culture, the cell sheets were formed, and the morphology of the cell sheets was then observed under a microscope (Olympus Corp.). For hematoxylin and eosin (H\&E) staining (Beijing Solarbio Science \& Technology Co., Ltd.), the cell sheets of each group were uncovered by cell scraping and fixed with $4 \%$ paraformaldehyde solution. 
Table I. Sequences of primers used for RT-qPCR.

\begin{tabular}{lll}
\hline Genes & \multicolumn{1}{c}{ Forward sequence } & \multicolumn{1}{c}{ Reverse sequence } \\
\hline GAPDH & 5'-GGAGCGAGATCCCTCCAAAAT-3' & 5'-GGCTGTTGTCATACTTCTCATGG-3' \\
ALP & 5'-GTGAACCGCAACTGGTACTC-3' & 5'-GAGCTGCGTAGCGATGTCC-3' \\
COL1 & 5'-GCTGATGATGCCAATGTGGTT-3' & 5'-CCAGTCAGAGTGGCACATCTTG-3' \\
RUNX2 & 5'-GTTTCACCTTGACCATAACCGT-3' & 5'-GGGACACCTACTCTCATACTGG-3' \\
OPN & 5'-CAGTTGTCCCCACAGTAGACAC-3' & 5'-GTGATGTCCTCGTCTGTAGCATC-3'
\end{tabular}

RT-qPCR, reverse transcription-quantitative polymerase chain reaction; R, reverse primer; F, forward primer; ALP, alkaline phosphatase; COL1, collagen type I; RUNX2, runt-related transcription factor 2; OPN, osteopontin.

Following routine dehydration, paraffin embedding and serial sections, H\&E staining was performed for $1 \mathrm{~h}$ at room temperature. For observation through scanning electron microscopy (SEM) (Phenom G2 Pro, Holland), the sheets were fixed with $3 \%$ glutaraldehyde ( $\mathrm{pH} 7.4$ ), dehydrated, critical point dried and sprayed with gold, and then the extension of PDLSCs in the sheets was observed.

Alkaline phosphatase (ALP) activity assay and ALP staining. Following the formation of cell sheets, osteogenic induction medium with various rutin concentrations was used for 7 days, and the cell sheets were then fixed with $4 \%$ paraformaldehyde for $30 \mathrm{~min}$ at room temperature. ALP staining was performed with an alkaline phosphatase staining kit (Nanjing Jiancheng Bioengineering Institute) for $15 \mathrm{~min}$ at room temperature. The sheets were washed 3 times with distilled water and observed under an inverted microscope (IX73; Olympus Corp.). For ALP activity detection, following 7 days of osteogenic induction, the cells were rinsed with PBS, lysed with RIPA buffer (Beyotime Institute of Biotechnology) and centrifuged at $4^{\circ} \mathrm{C}$ and $12,000 \mathrm{x} \mathrm{g}$ for $15 \mathrm{~min}$. According to the manufacturer's protocol of the ALP kit, the reaction solution was added to each test well. Subsequently, after $30 \mathrm{~min}$, a spectrophotometer (SPECTROstar Nano) was used to detect the absorbance value (OD value) at $520 \mathrm{~nm}$.

Alizarin Red staining and Alizarin Red semi-quantitative detection. Following the formation of cell sheets, the cells were cultured in osteogenic induction medium for 14 days and then fixed in $4 \%$ paraformaldehyde for $30 \mathrm{~min}$, followed by staining with Alizarin Red solution for $30 \mathrm{~min}$ at room temperature. Subsequently, the cells were washed with PBS, dried at room temperature and observed under an inverted microscope. Finally, mineralized nodules were dissolved in $2 \%$ cetylpyridinium chloride (Sigma-Aldrich; Merck KGaA) and OD values were measured at $562 \mathrm{~nm}$ using a spectrophotometer (SPECTROstar Nano) for statistical analysis.

Reverse transcription-quantitative PCR (RT-qPCR). PDLSCs (1) $10^{5} /$ well) were seeded into 6 -well paltes. Following cell adherence, the culture medium was replaced with sheet culture medium. Following 10 days of sheet formation, the formed cell sheets was further induced in osteogenic induction medium, and the osteogenic genes were then detected by RT-qPCR on days 7 and 14. This time point of detecting osteogenic genes was selected based on the published literature $(14,20,37)$. Total RNA was extracted using TRIzol ${ }^{\circledR}$ reagent (Takara Bio, Inc.) according to the manufacturer's protocol. A total of $1 \mu \mathrm{g}$ RNA was reverse transcribed into complementary DNA (cDNA) using a RevertAid First Strand cDNA Synthesis kit (Thermo Fisher Scintific, Inc). qPCR was performed using a Roche Light Cycler ${ }^{\circledR} 480 I I$. The qPCR parameters used were as follows: $95^{\circ} \mathrm{C}$ for $15 \mathrm{sec}, 40$ cycles of $95^{\circ} \mathrm{C}$ for $5 \mathrm{sec}$, followed by $60^{\circ} \mathrm{C}$ for $60 \mathrm{sec}$. Relative gene expression data was calculated through the $2^{-\Delta \Delta \mathrm{Cq}}$ method (38). The gene primers used are listed in Table I. GAPDH was used for normalization.

Western blot analysis. The PDLSCs were seeded into 6-well plates at a density of $1 \times 10^{5} /$ well. Following 10 days of sheet formation, total protein was extracted to detect the expression of ECM-related proteins. Following the formation of the sheets, cells were cultured in osteogenic induction medium for 7 or 14 days. Briefly, the sheets in the 6-well plate were scraped off using a cell scraper and transferred to sterile tubes. Cells were lysed using RIPA buffer (Beyotime Institute of Biotechnology), centrifuged at $4^{\circ} \mathrm{C}$ and $12,000 \mathrm{x}$ g for $15 \mathrm{~min}$, and the supernatant was removed and transferred to different sterile tubes. The total protein of each group was extracted and the protein concentration was determined using a BCA Protein assay kit (Beijing Solarbio Science \& Technology Co., Ltd.). Subsequently, 10\% SDS-PAGE was performed with a suitable amount of protein. The protein was transferred to a PVDF membrane (EMD Millipore), blocked with 5\% skim milk powder, and incubated with primary antibodies overnight at $4^{\circ} \mathrm{C}$. The following day, the membrane was then incubated with the secondary antibody (goat anti-rabbit IgG, cat. no. 7074S; Santa Cruz Biotechnology, Inc.) for $1 \mathrm{~h}$ at room temperature. The following primary antibodies were used: Anti-human GAPDH monoclonal (1:20,000, cat. no. HRP-60004; ProteinTech Group, Inc.), rabbit anti-human ALP (1:10,000, cat. no. ab108337; Abcam), rabbit anti-human osteopontin (OPN; 1:500, cat. no. ab8448; Abcam), rabbit anti-human runt-related transcription factor 2 (RUNX2; 1:1,000, cat. no. cst12556; Cell Signaling Technology), rabbit anti-human collagen type I (COL1; 1:500, cat. no. w10088; Wanlei Biotechnology Co., Ltd.), rabbit anti-human fibronectin (1:500, cat. no. wl00712; Wanlei Biotechnology Co., Ltd.), and rabbit anti-human integrin $\beta 1$ (1:500, cat. no. wl01615; Wanlei Biotechnology Co., Ltd.). The protein bands were visualized using enhanced chemiluminescence reagents (EMD 
A

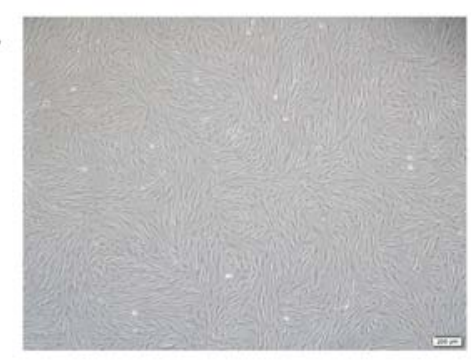

B

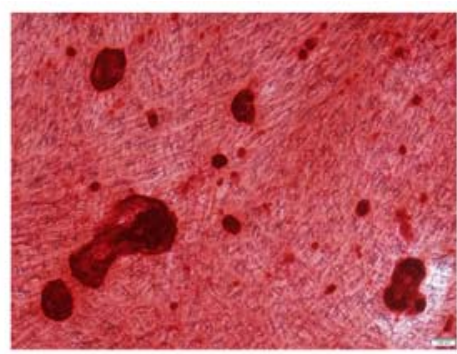

C

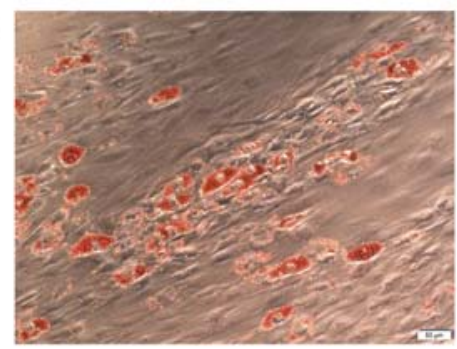

D

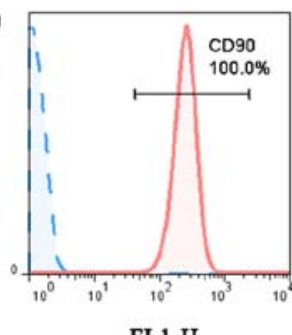

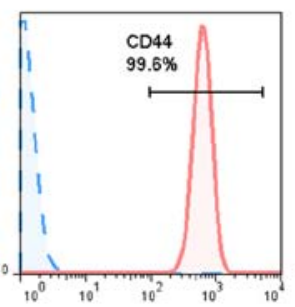

FL2-H

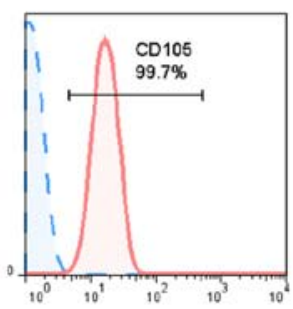

FL-3H

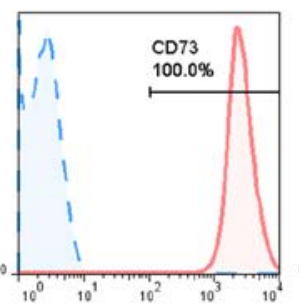

FL-4H

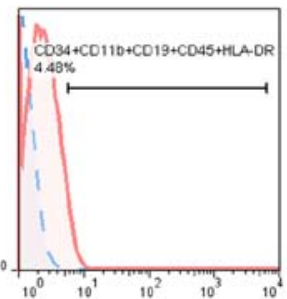

FL-2H

Figure 1. Characterization of PDLSCs. (A) PDLSCs presented a spindle-shaped morphology (scale bar, $200 \mu \mathrm{m}$ ). (B) Osteogenic differentiation of PDLSCs was demonstrated as red mineralized nodules (scale bar, $100 \mu \mathrm{m}$ ). (C) Adipogenic differentiation of PDLSCs was demonstrated as red oil drops (scale bar, $50 \mu \mathrm{m}$ ). (D) PDLSCs were positive for CD90, CD44, CD105, CD73 and negative for CD34, CD11b, CD19, CD45 and HLA-DR. PDLSC, periodontal ligament stem cell.

Millipore) and protein levels were analyzed using Image J (1.47V; National Institutes of Health).

Statistical analysis. The experimental data are expressed as the means \pm standard deviation, and were analyzed using SPSS17.0 software. A paired Student's t-test was used for comparisons between 2 groups, and one-way ANOVA with Tukey's post hoc test was used for comparisons between multiple groups. A value of $\mathrm{P}<0.05$ was considered to indicate a statistically significant difference.

\section{Results}

Isolation and identification of PDLSCs. Following 10 days of culture, the fusion degree of the cultured cells reached $90 \%$. The cells were spindle-shaped, fibroblast-like and spiral-like (Fig. 1A). Following 28 days of osteogenic induction, scattered milky white sandy nodules at the bottom of the Petri dish were visible to the eye, which varied in size. Alizarin Red staining and microscopic observation demonstrated that the milky white nodules were stained red (Fig. 1B). Under the action of adipogenic induction solution, cell proliferation decreased and the cells gradually changed from a spindle-like shape to an ellipse shape. Furthermore, 4 weeks later, some of the cells had beaded adipogenic droplets in the cytoplasm, and the Oil Red O staining result was red (Fig. 1C). Flow cytometric analysis revealed that CD90 FITC, CD105 PerCP-Cy5.5, CD73 APC and CD44 PE were highly expressed, while CD34 PE, CD11b PE, CD19 PE, CD45 PE and HLA-DR PE were almost not expressed (Fig. 1D). Thus, PDLSCs were successfully obtained.

Cell proliferation. CCK-8 was used to determine the effects of rutin on the proliferation of PDLSCs. In the VC medium with a certain concentration $(20 \mu \mathrm{g} / \mathrm{ml})$, the PDLSCs were treated with various concentrations of rutin, which had different effects on the proliferation of the PDLSCs. The OD values of

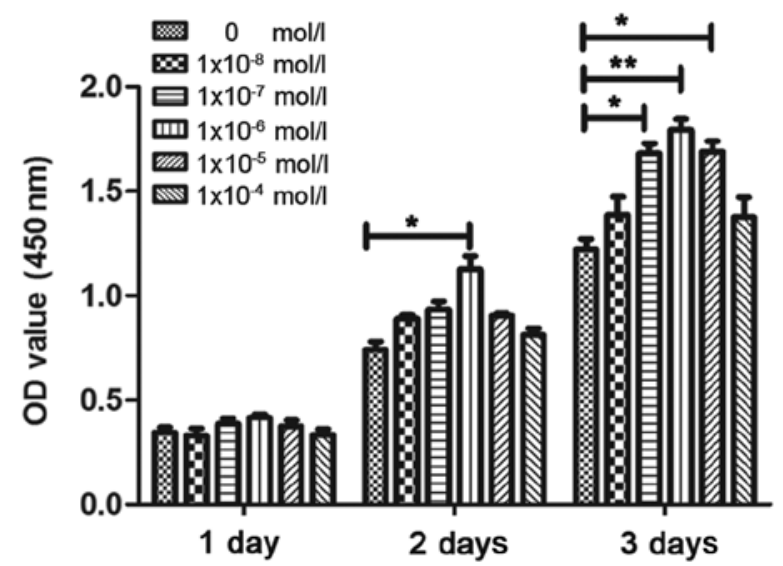

Figure 2. Rutin promotes the proliferation of PDLSCs. PDLSCs were cultured with various concentrations of rutin $\left(0,1 \times 10^{-8}, 1 \times 10^{-7}, 1 \times 10^{-6}, 1 \times 10^{-5}\right.$ and $\left.1 \times 10^{-4} \mathrm{~mol} / \mathrm{l}\right)$ in $\alpha$-minimum essential medium containing a certain concentration of VC $(20 \mu \mathrm{g} / \mathrm{ml})$ for 3 days. The OD value in cell induced with rutin at $1 \times 10^{-5}, 1 \times 10^{-6}$ and $1 \times 10^{-7} \mathrm{~mol} / 1$ was higher than that of the control group on day 3 and rutin at $1 \times 10^{-6} \mathrm{~mol} / 1$ resulted in the highest activity of proliferation among all groups. ${ }^{*} \mathrm{P}<0.05,{ }^{* *} \mathrm{P}<0.01$. PDLSC, periodontal ligament stem cell; VC, vitamin C.

rutin at concentrations of $1 \times 10^{-5}, 1 \times 10^{-6}$ and $1 \times 10^{-7} \mathrm{~mol} / \mathrm{l}$ were higher compared with those of the control group, which demonstrated that all 3 concentrations promoted cell proliferation; however, the $1 \times 10^{-6} \mathrm{~mol} / 1$ group promoted the proliferation of PDLSC sheets most significantly (Figs. 2 and S1A). Therefore, $1 \times 10^{-6} \mathrm{~mol} / \mathrm{l}$ rutin was determined to be the optimal concentration for the culture of cell sheets.

Morphological observation of cell sheets. Following 10 days of continuous culture, milky white membrane-like substances were observed at the bottom of the Petri dish in all groups of PDLSCs. The cell sheets of each group were carefully peeled off along the petri dish. It was found that the cell sheets were membrane-like, with a certain thickness and 
A

VC
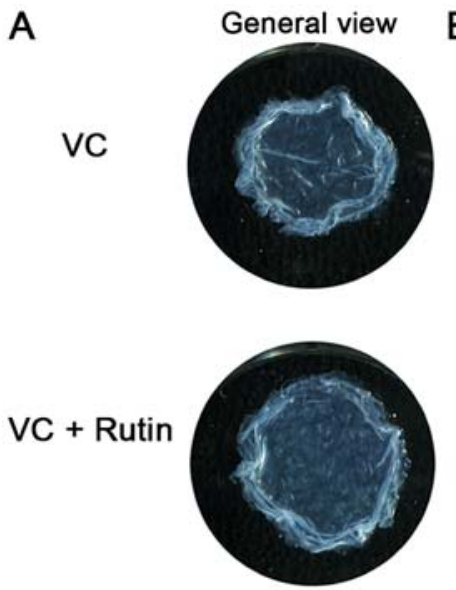

D

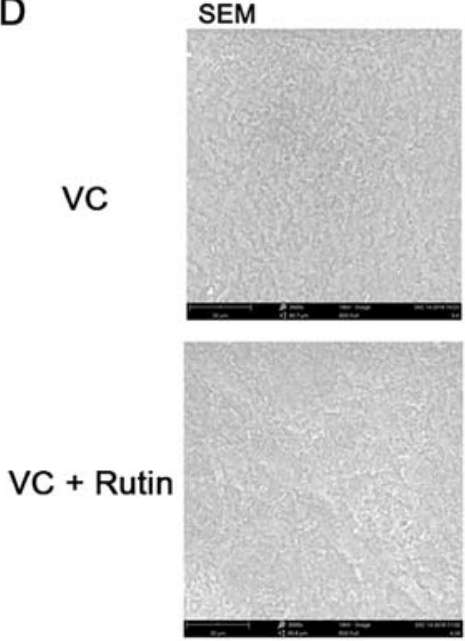

$x 2000$

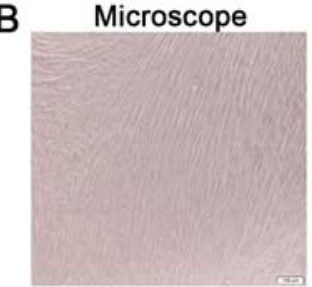

C
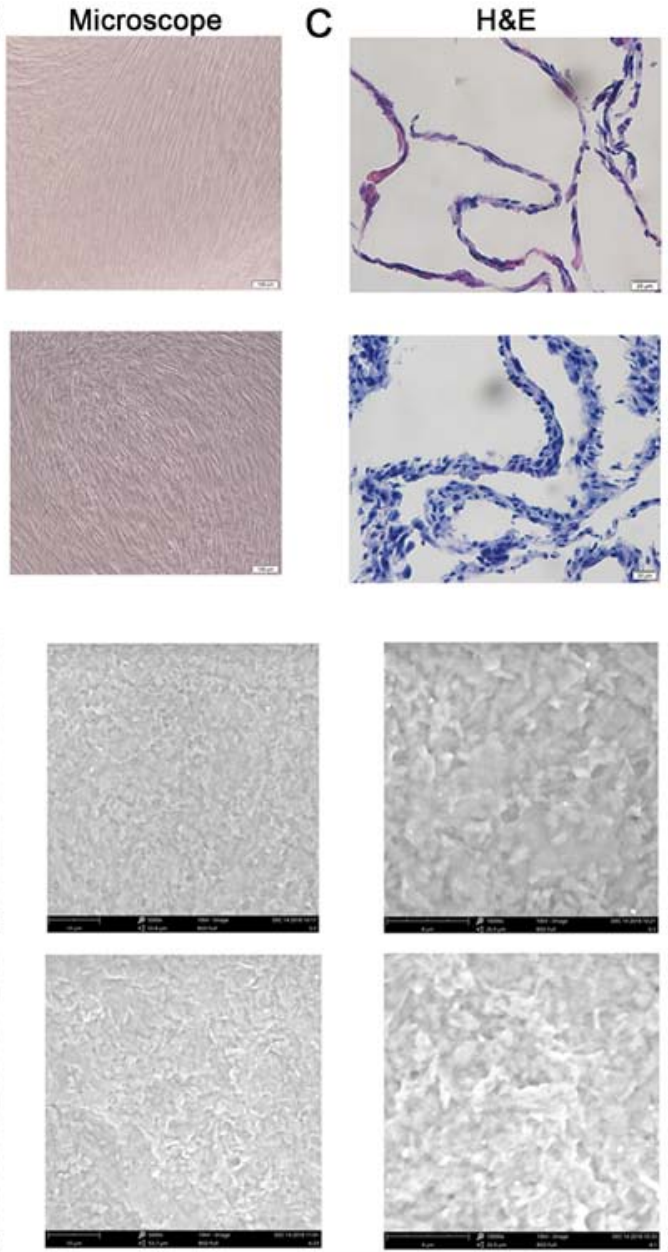

$\times 5000$
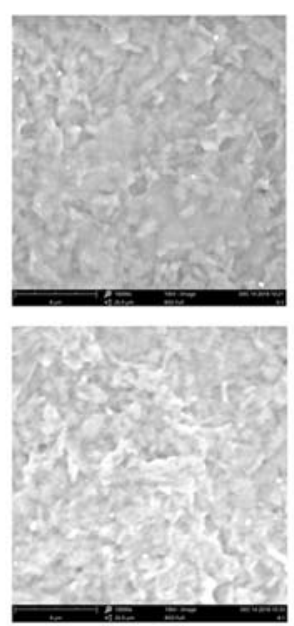

$\mathrm{x} 10000$

Figure 3. Morphological observation of cell sheets. Morphologies of the VC-induced PDLSC sheets and the rutin and VC co-cultured PDLSC sheets. (A) Representative images of cell sheets in culture dishes. (B) Morphologies of cell sheets were observed using an inverted microscope. (C) Morphologies of cell sheets were analyzed by hematoxylin and eosin staining. (D) Representative images of surface were observed by scanning electron microscopy. VC, vitamin C; PDLSC, periodontal ligament stem cell; H\&E, hematoxylin and eosin.

toughness (Fig. 3A). Under the inverted microscope, the cells in the rutin-induced group were denser and thicker (Figs. 3B and S1B). The results of H\&E staining revealed that the cell membrane was uniform and the nucleus was stained blue. There was abundant powdered ECM between cells. In addition, the results revealed that the cell sheet of the rutin-induced group consisted of 3-4 layers, while that of the VC-induced group consisted of 1-2 layers (Fig. 3C). The results of SEM revealed that in the rutin-induced group, the cell sheet cells arranged more closely and orderly, and the cells extended well and had abundant ECM (Fig. 3D).

Expression of ECM-related proteins in PDLSC sheets. Following 10 days of induction, the cell sheets were formed in the rutin and control groups. The results of western blot analysis revealed that the protein expression of fibronectin, integrin $\beta 1$ and COL1 in the PDLSC sheet was higher in the experimental group compared with the control group (Fig. 4). Following the addition of rutin, the expression of ECM-related proteins was increased.

Effects of rutin on the osteogenesis of PDLSC sheets. In order to further detect the osteogenic effects of rutin on
PDLSC sheets, the PDLSCs were induced in 6-well plates for 10 days with a fixed concentration of $20 \mu \mathrm{g} / \mathrm{ml} \mathrm{VC}$ and $10^{-6} \mathrm{~mol} / \mathrm{l}$ rutin, and were then induced with various concentrations of rutin. After 7 days, ALP staining and ALP activity assay were performed. After 14 days, Alizarin Red staining and Alizarin Red semi-quantitative assay were performed. ALP staining (Fig. 5A) and the ALP activity assay (Fig. 5C) revealed that $1 \times 10^{-6} \mathrm{~mol} / 1$ rutin significantly increased the ALP activity in PDLSC sheets. The results of Alizarin Red staining (Fig. 5B) and Alizarin Red semi-quantitative assay (Fig. 5D) revealed that the rutin-induced group exhibited an increase in mineralized nodules, and that rutin at $1 \times 10^{-6} \mathrm{~mol} / 1$ demonstrated the most potent effect. These results suggest that rutin promotes the osteogenic differentiation of PDLSC sheets, and that rutin at $1 \times 10^{-6} \mathrm{~mol} / 1$ promotes the osteogenic differentiation of PDLSC sheets most significantly.

Osteogenic-related gene and protein expression. The expression of osteogenic-related genes and proteins were detected by RT-qPCR and western blot analysis at 7 and 14 days following osteogenic induction. The results revealed that the protein (Fig. 6A-C) and mRNA (Fig. 6D and E) expression levels of 

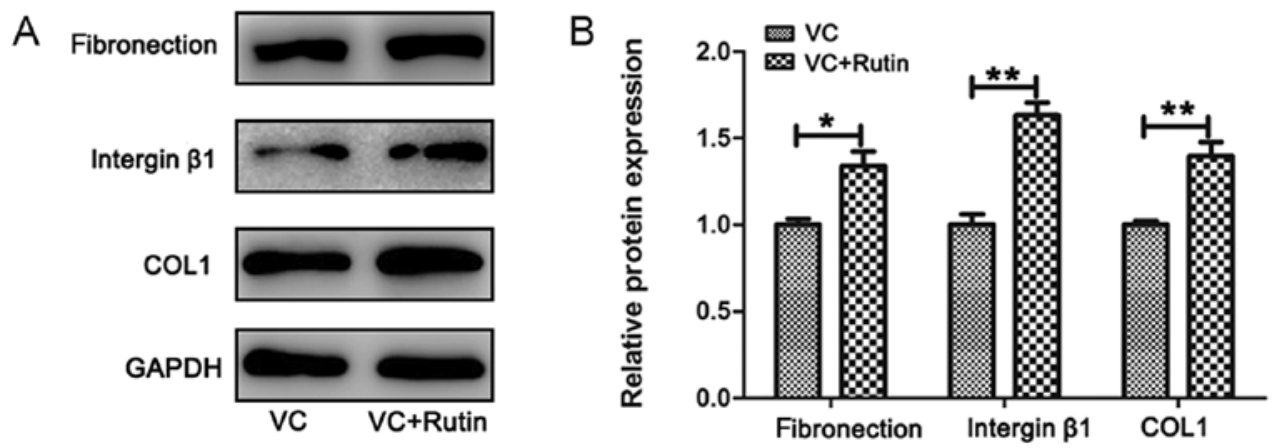

Figure 4. Expression of extracellular matrix-related proteins in PDLSC sheets. Following the formation of the cell sheets in the rutin and control groups, the protein expression of fibronectin, integrin $\beta 1$ and COL1 in PDLSC sheets was higher in the rutin group compared with the control group. (A) The protein expression of fibronectin, integrin $\beta 1$ and COL1 in PDLSC sheets of the rutin and control groups. (B) Relative quantitative analysis of fibronectin, integrin $\beta 1$ and COL1 proteins. ${ }^{*} \mathrm{P}<0.05,{ }^{* *} \mathrm{P}<0.01$. PDLSC, periodontal ligament stem cell; COL1, collagen, type I; VC, vitamin C.

A

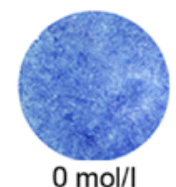

B

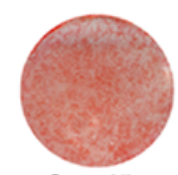

$0 \mathrm{~mol} / \mathrm{l}$
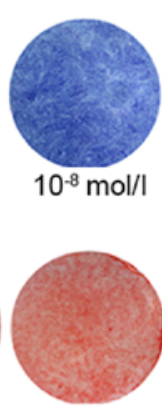

$10^{-8} \mathrm{~mol} / 1$

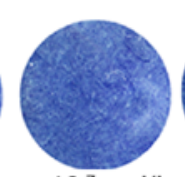

$10^{-7} \mathrm{~mol} / \mathrm{l}$

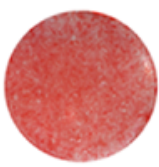

$10^{-7} \mathrm{~mol} / \mathrm{l}$
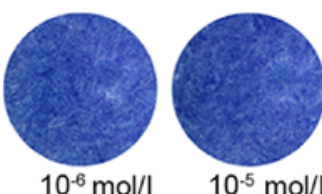

$10^{-5} \mathrm{~mol} /$
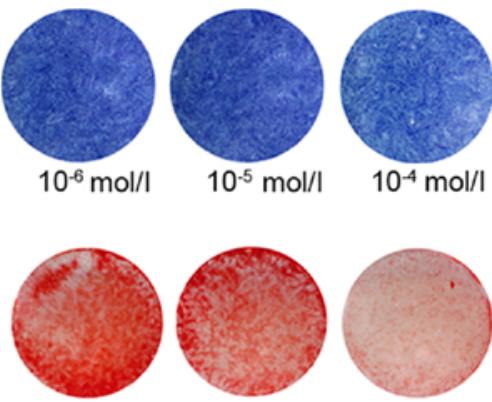

$10^{-6} \mathrm{~mol} / \mathrm{l}$

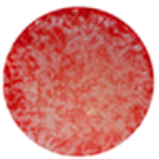

$10^{-5} \mathrm{~mol} / \mathrm{l}$

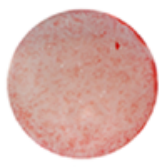

$10^{-4} \mathrm{~mol} / \mathrm{I}$
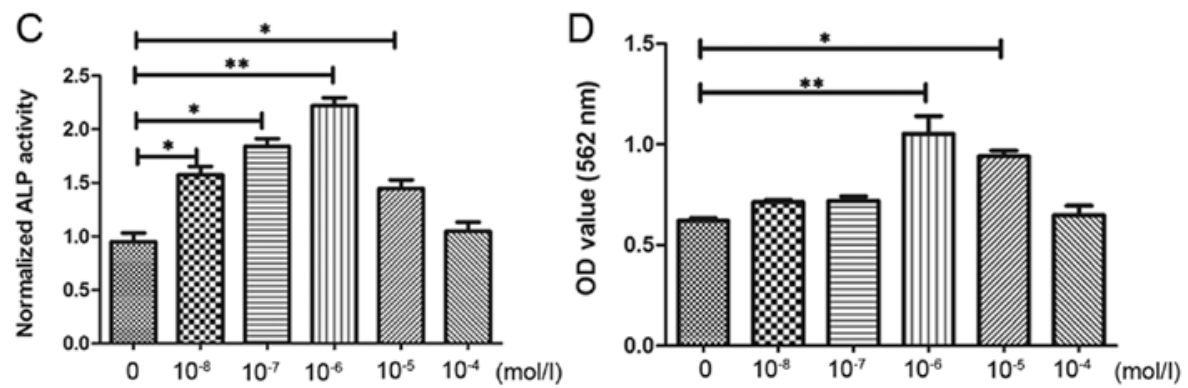

Figure 5. Rutin promotes the osteogenic differentiation of PDLSC cell sheets. Following the formation of cell sheets, PDLSCs were cultured with various concentrations of rutin $\left(0,1 \times 10^{-8}, 1 \times 10^{-7}, 1 \times 10^{-6}, 1 \times 10^{-5}\right.$ and $\left.1 \times 10^{-4} \mathrm{~mol} / \mathrm{l}\right)$ in osteogenic induction medium. It was found that $1 \times 10^{-6} \mathrm{~mol} / 1 \mathrm{rutin}$ significantly increased the ALP activity in the PDLSC sheets. (A) Following osteogenic induction for 7 days, ALP staining was performed. (B) Following osteogenic induction for 14 days, Alizarin Red staining was performed. (C) Result of the ALP activity assay following osteogenic induction for 7 days. (D) Result of Alizarin Red semi-quantitative analysis after osteogenic induction for 14 days. ${ }^{*} \mathrm{P}<0.05,{ }^{* *} \mathrm{P}<0.01$. PDLSC, periodontal ligament stem cell; ALP, alkaline phosphatase.

COL1, ALP, RUNX2, and OPN were higher in rutin-induced group compared with the control group.

\section{Discussion}

In recent years, cell sheet technology has widely been used in periodontal tissue regeneration and has achieved good results $(39,40)$. In the present study, we designed rutin-modified PDLSC sheets for periodontal tissue regeneration in vitro. First, PDLSCs were isolated and identified according to previously published articles $(14,37,41)$. Multilineage differentiation potential is one of the distinguishing features of MSCs. Thus far, a number of studies have used the osteogenic induction method and adipogenic induction method to detect the multiple differentiation ability of MSCs $(36,42)$. In the current study, the ability of PDLSCs to differentiate into osteoblasts was demonstrated by Alizarin Red staining. On the other hand, the ability of PDLSCs to differentiate into adipocytes was demonstrated by Oil Red O staining. In flow cytometric analysis, the isolated cells highly expressed CD90 FITC, CD105 PerCP-Cy5.5, CD73 APC and CD44 PE, and almost did not express CD34 PE, CD11b PE, CD19PE, CD45 PE and HLA-DR PE, which was consistent with the standards formulated by the International Society for Cellular Therapy (43). Additionally, the results of the present study were consistent with those of previous studies $(35,42,44)$. Thus, the present study successfully obtained PDLSCs.

Developing and optimizing cell sheet technology can help promote periodontal regeneration via stem-cell therapy. In order to obtain more efficient cell sheets, bioactive substances can be added to the sheet culture medium to promote the function of PDLSCs. Previous studies have demonstrated that rutin 
A

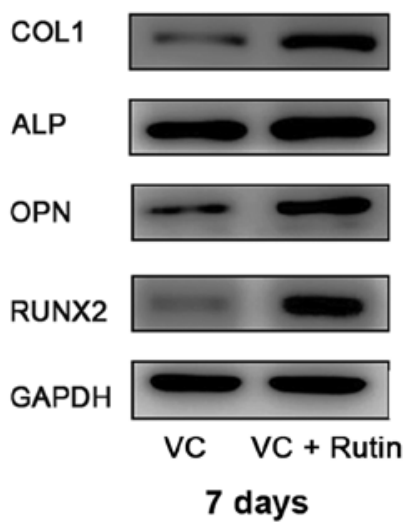

B

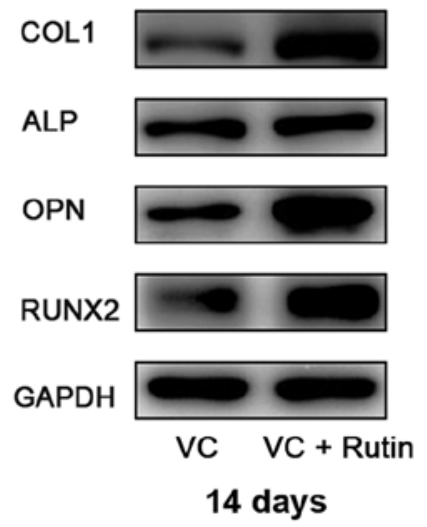

C
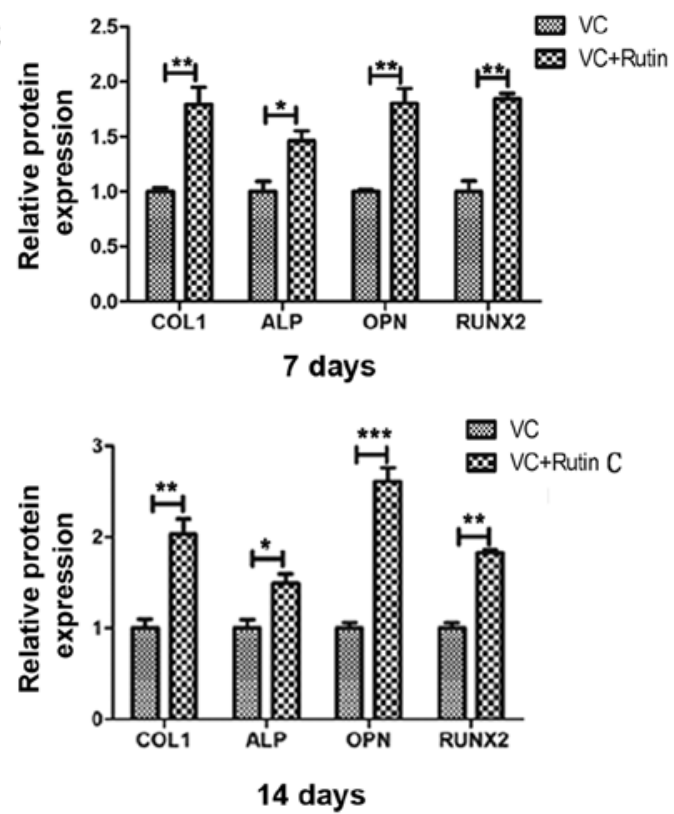

D

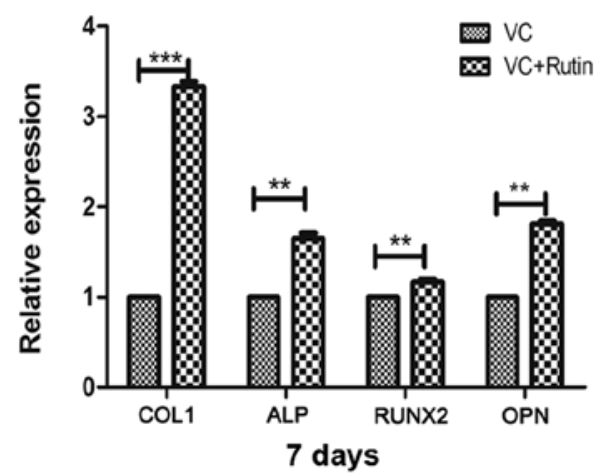

$\mathrm{E}$

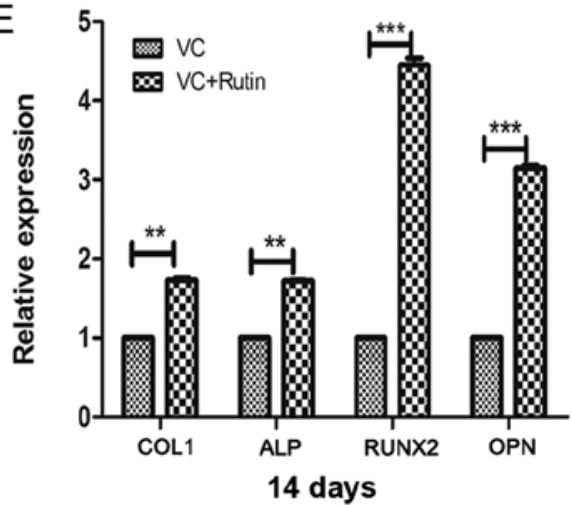

Figure 6. Osteogenic gene and protein expression. The protein and mRNA expression of COL1, ALP, OPN and RUNX2 in PDLSC sheets of the rutin and control groups following osteogenic induction for 7 and 14 days. The protein expression of COL1, ALP, RUNX2 and OPN after osteogenic induction for (A) 7 days and (B) 14 days. (C) Relative quantitative analysis of COL1, ALP, RUNX2 and OPN proteins on days 7 and 14 . The mRNA expression of COL1, ALP, RUNX2 and OPN after osteogenic induction for (D) 7 days and (E) 14 days. ${ }^{*} \mathrm{P}<0.05,{ }^{* *} \mathrm{P}<0.01,{ }^{* * *} \mathrm{P}<0.001$. PDLSC, periodontal ligament stem cell; COL1, collagen, type I; ALP, alkaline phosphatase; RUNX2, runt-related transcription factor 2; OPN, osteopontin; VC, vitamin C.

can enhance the effects of vitamin C $(31,45)$. Applying this characteristic to stem cell sheet formation may contribute to cultivating cell sheets. The results of the present study first confirmed that a combination of rutin at $1 \times 10^{-6} \mathrm{~mol} / 1$ and $\mathrm{VC}$ $(20 \mu \mathrm{g} / \mathrm{ml})$ effectively promoted the formation of PDLSC sheets, and the proliferation of PDLSCs was accelerated in the rutin-induced group. However, a perfect cell sheet not only needs to form rapidly, but also requires a certain thickness, toughness and abundant ECM. Therefore, H\&E staining, SEM observation and ECM-related protein detection were used to observe the effects of rutin on cell sheet formation. The results revealed that following 10 consecutive days of culture, the cells in each group grew in multiple layers. H\&E staining revealed that the number of cell layers in the control group was 1-2 layers, while the number of cell layers in the rutin-induced group was up to 3-4 layers. ECM was abundant in the cell sheets of the 2 cell groups, and the cell arrangement was regular. SEM observation revealed cell extension and cell-to-cell junctions in the 2 groups, and the surface structure of the rutin-induced cells was more compact. These results indicated that the addition of rutin further promoted the formation of cell sheets. In addition, the regulation of the ECM on cells depends on proteins in the ECM, which determine the shape of cells, control cell differentiation and participate in cell migration $(46,47)$. Firstly, fibronectin can promote the binding of cells to the matrix (48). COL1 and proteoglycan are the basic skeletons that form a fibrous reticular complex on the cell surface (49). The majority of the receptors are membrane integrins, which are connected to the cytoskeleton proteins in the cell. The ECM connects the extracellular and intracellular regions via integrin, which is conducive to the transmission of intracellular and extracellular signals (50). Therefore, the expression of the ECM-related proteins, COL1, fibronectin and integrin $\beta 1$, was observed in the present study. The results revealed that rutin promoted the expression of fibronectin, COL1 and integrin $\beta 1$.

In order to further examine whether rutin can improve the osteogenic efficiency of stem cell sheets, the expression of osteogenic-related genes was investigated. Osteoblasts predominantly express COL1, ALP, RUNX2, OPN and other ECM-related proteins during cell proliferation, matrix maturation and mineralization stages. Observing the expres- 
sion of these marker genes and protein levels can indicate the differentiation and maturation stage of MSCs into osteoblasts to a certain extent. Previous studies have indicated that ALP and RUNX2 are secreted in the early stages of osteogenic differentiation, and OPN is a necessary factor for bone calcification and mineralization (51). The results of the present study demonstrated that rutin increased the expression of ALP, RUNX2 and OPN in the cell sheets. Furthermore, ALP staining, the ALP activity assay, Alizarin Red staining and the Alizarin Red semi-quantitative results confirmed that rutin enhanced the osteogenic differentiation ability of PDLSC sheets compared with those induced with VC alone. Therefore, rutin and vitamin $\mathrm{C}$ may exert a more significant effect in promoting the formation and osteogenic differentiation of cell sheets. The present experimental results confirmed that rutin, a bioflavonoid, can effectively enhance the effects of $\mathrm{VC}$ and accelerate the formation of cell sheets. This induction method may provide higher quality and more effective cell sheets.

In conclusion, the results of the present study demonstrated that rutin, a natural bioflavonoid, promotes the formation of PDLSC sheets and bone regeneration, which is expected to become an important tool in the improvement and optimization of cell sheet technology. The method based on ruitn and VC co-treatment is effective and potentially valuable. Therefore, the present study provides a theoretical basis for the future application of periodontal ligament stem cell sheet in the study of periodontal tissue regeneration, and provides a novel proposition for tissue engineering to regenerate periodontal tissue.

\section{Acknowledgements}

The authors would like to thank the Director of Shandong Provincial Key Laboratory of Oral Tissue Regeneration for providing technical support.

\section{Funding}

The present study was supported by the Construction Engineering Special Fund of Taishan Scholars (grant no. ts201511106).

\section{Availability of data and materials}

All data generated or analyzed during this study are included in this published article or are available from the corresponding author on reasonable request.

\section{Authors' contributions}

XX designed the experiments. BZ wrote the manuscript. BZ and YX performed the experiments. BZ and YZ analyzed the data. All authors have read and approved the final manuscript.

\section{Ethics approval and consent to participate}

This study was approved by the Medical Ethical Committee of School of Stomatology, Shandong University (Shandong, China). Informed consent was obtained in writing by all donors and their parents.

\section{Patient consent for publication}

Not applicable.

\section{Competing interests}

The authors declare that they have no competing interests.

\section{References}

1. Scapoli L, Girardi A, Palmieri A, Martinelli M, Cura F, Lauritano D and Carinci F: Quantitative analysis of periodontal pathogens in periodontitis and gingivitis. J Biol Regul Homeost Agents 29 (Suppl 1): 101-110, 2015.

2. Chen FM, Zhang J, Zhang M, An Y, Chen F and Wu ZF: A review on endogenous regenerative technology in periodontal regenerative medicine. Biomaterials 31: 7892-7927, 2010.

3. Chen FM and Jin Y: Periodontal tissue engineering and regeneration: Current approaches and expanding opportunities. Tissue Eng Part B Rev 16: 219-255, 2010.

4. Matsuda N, Shimizu T, Yamato Mand Okano T: Tissue Engineering Based on Cell Sheet Technology. Adv Mater 19: 3089-3099, 2007.

5. Nakamura A, Akahane M, Shigematsu H, Tadokoro M, Morita Y, Ohgushi H, Dohi Y, Imamura T and Tanaka Y: Cell sheet transplantation of cultured mesenchymal stem cells enhances bone formation in a rat nonunion model. Bone 46: 418-424, 2010.

6. Sukho P, Cohen A, Hesselink JW, Kirpensteijn J, Verseijden F and Bastiaansen-Jenniskens YM: Adipose Tissue-Derived Stem Cell Sheet Application for Tissue Healing In Vivo: A Systematic Review. Tissue Eng Part B Rev 24: 37-52, 2018.

7. Matsuura K, Utoh R, Nagase K and Okano T: Cell sheet approach for tissue engineering and regenerative medicine. J Control Release 190: 228-239, 2014.

8. Miyagawa S, Saito A, Sakaguchi T, Yoshikawa Y, Yamauchi T, Imanishi Y, Kawaguchi N, Teramoto N, Matsuura N, Iida H, et al: Impaired myocardium regeneration with skeletal cell sheets - a preclinical trial for tissue-engineered regeneration therapy. Transplantation 90: 364-372, 2010.

9. Itaba N, Matsumi Y, Okinaka K, Ashla AA, Kono Y, Osaki M, Morimoto M, Sugiyama N, Ohashi K, Okano T, et al: Human mesenchymal stem cell-engineered hepatic cell sheets accelerate liver regeneration in mice. Sci Rep 5: 16169, 2015.

10. Nishida K, Yamato M, Hayashida Y, Watanabe K, Yamamoto K, Adachi E, Nagai S, Kikuchi A, Maeda N, Watanabe H, et al: Corneal reconstruction with tissue-engineered cell sheets composed of autologous oral mucosal epithelium. N Engl J Med 351: 1187-1196, 2004.

11. Shimizu K, Ito A, Yoshida T, Yamada Y, Ueda M and Honda H: Bone tissue engineering with human mesenchymal stem cell sheets constructed using magnetite nanoparticles and magnetic force. J Biomed Mater Res B Appl Biomater 82: 471-480, 2007.

12. Tsumanuma Y, Iwata T, Washio K, Yoshida T, Yamada A, Takagi R, Ohno T, Lin K, Yamato M, Ishikawa I, et al: Comparison of different tissue-derived stem cell sheets for periodontal regeneration in a canine 1-wall defect model. Biomaterials 32: 5819-5825, 2011.

13. Okano T, Yamada N, Okuhara M, Sakai H and Sakurai Y: Mechanism of cell detachment from temperature-modulated, hydrophilic-hydrophobic polymer surfaces. Biomaterials 16: 297-303, 1995.

14. Wang Z, Feng Z, Wu G, Bai S, Dong Y and Zhao Y: In vitro studies on human periodontal ligament stem cell sheets enhanced by enamel matrix derivative. Colloids Surf B Biointerfaces 141: 102-111, 2016.

15. Akahane M, Shimizu T, Kira T, Onishi T, Uchihara Y, Imamura T and Tanaka Y: Culturing bone marrow cells with dexamethasone and ascorbic acid improves osteogenic cell sheet structure. Bone Joint Res 5: 569-576, 2016.

16. Weber P, Bendich A and Schalch W: Vitamin C and human health - a review of recent data relevant to human requirements. Int J Vitam Nutr Res 66: 19-30, 1996.

17. Naidu KA: Vitamin $C$ in human health and disease is still a mystery? An overview. Nutr J 2: 7-7, 2003.

18. Zhang Y, Sun GF, Jin YP, Jia LH and Wang Y: Effects of fluoride on proliferation and differentiation of rat osteoblasts in vitro and the antagonistic action of vitamin C. Zhonghua Lao Dong Wei Sheng Zhi Ye Bing Za Zhi 21: 250-252, 2003 (In Chinese). 
19. Van Pham P, Tran NY, Phan NL, Vu NB and Phan NK: Vitamin C stimulates human gingival stem cell proliferation and expression of pluripotent markers. In Vitro Cell Dev Biol Anim 52: 218-227, 2016.

20. Wei F, Qu C, Song T, Ding G, Fan Z, Liu D, Liu Y, Zhang C, Shi $S$ and Wang S: Vitamin C treatment promotes mesenchymal stem cell sheet formation and tissue regeneration by elevating telomerase activity. J Cell Physiol 227: 3216-3224, 2012.

21. Zhou S, Zou Q, Zhang K, Yang R, Zhao W and Fu Q: A Novel Experimental Study on Establish a Myoblasts Differentiated Cell Sheet Using Induced Adipose-Derived Stem Cell Technology. J Biomater Tissue Eng 7: 371-378, 2017.

22. Zhang G, Wang Z and Yan $H$ : The relationship between chemical structure and bioactivity of bioflavonoids. J Biol 22: 4-7, 2005.

23. Sharma S, Ali A, Ali J, Sahni JK and Baboota S: Rutin: Therapeutic potential and recent advances in drug delivery. Expert Opin Investig Drugs 22: 1063-1079, 2013.

24. La Casa C, Villegas I, Alarcón de la Lastra C, Motilva V and Martín Calero MJ: Evidence for protective and antioxidant properties of rutin, a natural flavone, against ethanol induced gastric lesions. J Ethnopharmacol 71: 45-53, 2000

25. Guardia T, Rotelli AE, Juarez AO and Pelzer LE: Anti-inflammatory properties of plant flavonoids. Effects of rutin, quercetin and hesperidin on adjuvant arthritis in rat. Farmaco 56: 683-687, 2001.

26. Diwan V, Brown L and Gobe GC: The flavonoid rutin improves kidney and heart structure and function in an adenine-induced rat model of chronic kidney disease. J Funct Foods 33: 85-93, 2017.

27. Piller NB: A comparison of the effectiveness of some anti-inflammatory drugs on thermal oedema. Br J Exp Pathol 56: 554-560, 1975.

28. Hosseinzadeh $\mathrm{H}$ and Nassiri-Asl M: Review of the protective effects of rutin on the metabolic function as an important dietary flavonoid. J Endocrinol Invest 37: 783-788, 2014.

29. Hyun H, Park H, Jeong J, Kim J, Kim H, Oh HI, Hwang HS and Kim HH: Effects of Watercress Containing Rutin and Rutin Alone on the Proliferation and Osteogenic Differentiation of Human Osteoblast-like MG-63 Cells. Korean J Physio Pharmacol 18: 347-352, 2014.

30. Crampton EW and Lloyd LE: A quantitative estimation of the effect of rutin on the biological potency of vitamin C. J Nutr 41 : 487-498, 1950

31. Guo R, Wei P and Liu W: Combined antioxidant effects of rutin and vitamin $\mathrm{C}$ in Triton X-100 micelles. J Pharm Biomed Anal 43: 1580-1586, 2007.

32. Alsaif MA: Beneficial effects of rutin and vitamin $\mathrm{C}$ coadministration in a streptozotocin-induced diabetes rat model of kidney nephrotoxicity. Pak J Nutr 8: 745-754, 2009.

33. Alsaif MA: Combined treatment of rutin and vitamin $\mathrm{C}$ improves the antioxidant status in streptozotocin-induced diabetic rats. J Med Sci 9: 1-9, 2009.

34. Seo BM, Miura M, Gronthos S, Bartold PM, Batouli S, Brahim J, Young M, Robey PG, Wang CY and Shi S: Investigation of multipotent postnatal stem cells from human periodontal ligament. Lancet 364: 149-155, 2004

35. Wada N, Menicanin D, Shi S, Bartold PM and Gronthos S: Immunomodulatory properties of human periodontal ligament stem cells. J Cell Physiol 219: 667-676, 2009.

36. Yin L, Cheng W, Qin Z, Yu H, Yu Z, Zhong M, Sun K and Zhang W: Effects of Naringin on Proliferation and Osteogenic Differentiation of Human Periodontal Ligament Stem Cells In Vitro and In Vivo. Stem Cells Int 2015: 758706, 2015.

37. Li M, Feng C, Gu X, He Q and Wei F: Effect of cryopreservation on proliferation and differentiation of periodontal ligament stem cell sheets. Stem Cell Res Ther 8: 77, 2017.
38. Livak KJ and Schmittgen TD: Analysis of relative gene expression data using real-time quantitative PCR and the 2(-Delta Delta C(T)) Method. Methods 25: 402-408, 2001

39. Panduwawala CP, Zhan X, Dissanayaka WL, Samaranayake LP, Jin $L$ and Zhang $C$ : In vivo periodontal tissue regeneration by periodontal ligament stem cells and endothelial cells in three-dimensional cell sheet constructs. J Periodontal Res 52: 408-418, 2017.

40. Hu J, Cao Y, Xie Y, Wang H, Fan Z, Wang J, Zhang C, Wang J, Wu CT and Wang S: Periodontal regeneration in swine after cell injection and cell sheet transplantation of human dental pulp stem cells following good manufacturing practice. Stem Cell Res Ther 7: 130, 2016.

41. Kim JH, Ko SY, Lee JH, Kim DH and Yun JH: Evaluation of the periodontal regenerative properties of patterned human periodontal ligament stem cell sheets. J Periodontal Implant Sci 47: 402-415, 2017.

42. Deng C, Sun Y, Liu H, Wang W, Wang J and Zhang F: Selective adipogenic differentiation of human periodontal ligament stem cells stimulated with high doses of glucose. PLoS One 13: e0199603, 2018.

43. Dominici M, Le Blanc K, Mueller I, Slaper-Cortenbach I, Marini F, Krause D, Deans R, Keating A, Prockop Dj and Horwitz E: Minimal criteria for defining multipotent mesenchymal stromal cells. The International Society for Cellular Therapy position statement. Cytotherapy 8: 315-317, 2006.

44. Xing Y, Zhang Y, Jia L and Xu X: Lipopolysaccharide from Escherichia coli stimulates osteogenic differentiation of human periodontal ligament stem cells through Wnt/ $\beta$-catenin-induced TAZ elevation. Mol Oral Microbiol 34: 2019.

45. Je HD, Shin CY, Park SY, Yim SH, Kum C, Huh IH, Kim JH and Sohn UD: Combination of vitamin $\mathrm{C}$ and rutin on neuropathy and lung damage of diabetes mellitus rats. Arch Pharm Res 25: $184-190,2002$

46. Adams JC and Watt FM: Regulation of development and differentiation by the extracellular matrix. Development 117: 1183-1198, 1993.

47. Raines EW: The extracellular matrix can regulate vascular cell migration, proliferation, and survival: Relationships to vascular disease. Int J Exp Pathol 81: 173-182, 2000.

48. Thant AA, Nawa A, Kikkawa F, Ichigotani Y, Zhang Y, Sein TT, Amin AR and Hamaguchi M: Fibronectin activates matrix metalloproteinase-9 secretion via the MEK1-MAPK and the PI3K-Akt pathways in ovarian cancer cells. Clin Exp Metastasis 18: 423-428, 2000.

49. Soikkeli J, Podlasz P, Yin M, Nummela P, Jahkola T, Virolainen S, Krogerus L, Heikkilä P, von Smitten K, Saksela O, et al: Metastatic outgrowth encompasses COL-I, FN1, and POSTN up-regulation and assembly to fibrillar networks regulating cell adhesion, migration, and growth. Am J Pathol 177: 387-403, 2010.

50. Wilkins JA, Stupack D, Stewart S and Caixia S: Beta 1 integrin-mediated lymphocyte adherence to extracellular matrix is enhanced by phorbol ester treatment. Eur J Immunol 21: 517-522, 1991.

51. Deng Y, Wu S, Zhou H, Bi X, Wang Y, Hu Y, Gu P and Fan X: Effects of a miR-31, Runx2, and Satb2 regulatory loop on the osteogenic differentiation of bone mesenchymal stem cells. Stem Cells Dev 22: 2278-2286, 2013.

This work is licensed under a Creative Commons Attribution-NonCommercial-NoDerivatives 4.0 International (CC BY-NC-ND 4.0) License. 